\title{
Fatores de risco para a ocorrência de déficit estatural em pré-escolares
}

\author{
Risk factors for short stature \\ in preschool children
}

Lenir Vaz Guimarães 1

Maria do Rosário Dias de Oliveira Latorre 2

Marilisa Berti de Azevedo Barros 3

\footnotetext{
1 Instituto de Saúde Coletiva, Universidade Federal de Mato Grosso. Av. Fernando Corrêa da Costa s/nọ, CCBS III, Campus Universitário, Cuiabá, Mato Grosso, 78060-900, Brasil. Ivguima@zaz.com.br 2 Departamento de Epidemiologia, Faculdade de Saúde Pública, Universidade de São Paulo. Av. Dr. Arnaldo 715, São Paulo, SP 01246-904, Brasil. mdrddola@usp.br 3 Departamento de Medicina Preventiva e Social, Faculdade de Ciências Médicas, Universidade Estadual de Campinas. Campus Universitário Zeferino Vaz, Distrito Barão Geraldo, Campinas, SP, 13081-970, Brasil. abarros@correionet.com.br
}

Abstract A case-control study was conducted to know the magnitude of the effect of main risk factors for short stature of pre-school children in a city in São Paul o State, in 1995. An anthropometric survey carried out with all children (1201) attending public pre-school classes was used to select the case and the control groups. It was selected a random sample of 165 children among those with stature/age $\leq-1 \mathrm{Z}$ score (case) and another sample of 165 among those with stature/age $\geq+1 \mathrm{Z}$ score (control). The mothers or persons responsi ble for these children were interviewed in order to obtain information on demographic, maternal and socio-economic variables. The multiple logistic hierarchical analysis showed the following variables as associated with preschool children's short stature: mother's educational level $(\mathrm{OR}=2,1 ; \mathrm{Cl}: 1,1-3,8)$; per capita family income $\leq 0,5$ $\mathrm{SM}(\mathrm{OR}=3,4 ; \mathrm{Cl}: 1,5-8,0)$; number of persons in the house $\geq 6(\mathrm{OR}=3,7 ; \mathrm{Cl}: 1,5-9,0)$; number of domestic equipment $\leq 1(\mathrm{OR}=4,4 ; \mathrm{Cl}: 1,8-10,7)$; birth length $<48(\mathrm{OR}=7,4 ; \mathrm{Cl}: 2,3-23,7)$, mother's stature $\leq 156,6(\mathrm{OR}=5,9 ; \mathrm{Cl}: 3,1-11,0)$ and father's stature $(\mathrm{OR}=4,2 ; \mathrm{Cl}: 2,1-8,6)$. We was found that even in a population of preschool children without nutritional deficiency (as measured by the usual anthropometric index) it is possi ble to observethe effect of socio economic variables in children stature. Key words Nutritional Status; Anthropometry; Pre-School Child; Case-Control Studies

Resumo Um estudo caso-controle foi desenvol vido para identificar a magnitude do efeito dos principais fatores associados à estatura baixa de pré-escolares em uma cidade do interior paulista, no ano de 1995. Dentre os 1.201 pré escol ares que freqüentavam as escolas públicas do muni cípi o, selecionaram-se 165 crianças que apresentavam um índice estatura/idade $\leq-1$ escore $Z$ (casos) e 165 entre as que tinham estatura/idade $\geq 1$ escore $Z$ (controles). As mães ou os responsáveis pelas crianças foram entrevistados para a obtenção de informações sócio-econômi cas, características da família e características reproduti vas maternas e antropométricas. A análise de regressão logística múltipla hi erarquizada indicou maior chance de déficit estatural nos seguintes grupos de préescolares: com baixa escolaridade da mãe $(O R=2,1 ; I C 1,1-3,8)$, renda familiar per capita $\leq 0,50 \mathrm{SM}(\mathrm{OR}=3,4 ; \mathrm{IC}: 1,5-8,0)$, número de pessoas no domicílio $\geq 6$ (OR = 3,7; IC: 1,5-9,0), número de equi pamentos domésticos $\leq 1$ ( OR =4,4; IC: 1,8-10,7), comprimento ao nascer $<48 \mathrm{~cm}(\mathrm{OR}=7,4$; IC: 2,3-23,7), estatura da mãe $\leq 156,6 \mathrm{~cm}(\mathrm{OR}=5,9 ;$ IC: 3,1-11,0) e estatura do pai $\leq 169,8 \mathrm{~cm}(\mathrm{OR}=4,2 ; \mathrm{IC}: 2,1-8,6)$. Verificou-se que, mesmo em uma população de pré-escolares em que a deficiência nutricional é mínima ou inexistente (medida pelos índices habituais), é possível evidenciar o efeito de variáveis sócio-econômi cas na estatura das crianças. Palavras-chave Estado Nutricional; Antropometria; Pré-Escolar; Estudos de Casos eControles 
Introdução

Muitas investigações têm buscado aprofundar o conhecimento sobre os fatores econômicos, sociais e biológicos que interferem na estatura de crianças e adultos. $O$ efeito das condições sócio-econômicas sobre o crescimento infantil tem sido observado em diferentes contextos e apresentado grande magnitude (Bobák et al., 1994; Amigo \& Bustos, 1995). Variáveis relacionadas aos antecedentes reprodutivos da mãe e às características da família têm sido apontadas freqüentemente como fatores associados à estatura atingida pelas crianças (Goldstein, 1971; Lindgren \& Cernerud, 1992; Barros \& Barros, 1994).

Com relação às variáveis biológicas, tem sido encontrado que o retardo estatural é mais freqüente nos meninos do que nas meninas (Barros et al., 1990). Tanner (1987) refere que, com respeito ao retardo do crescimento linear, os meninos são, em geral, mais susceptíveis que as meninas às condições desfavoráveis de vida. Tem sido relatada também a associação entre o peso e o comprimento ao nascer com o crescimento posterior da criança (Olinto et al., 1993; Miller \& Korenman, 1994). A relação entre a estatura dos pais e a dos filhos é amplamente reconhecida na literatura (Goldstein, 1971; Guilliford et al., 1991; Amigo et al., 1997; Voss et al., 1998). Tendo em vista que a estatura dos pais expressa, em certo grau, o potencial biológico do crescimento da criança, o uso desta informação torna-se relevante para a análise da variabilidade do crescimento físico.

Como enfatiza Bicudo-Zeferino (1992:2), “o crescimento é a expressão da interação extremamente complexa entre o potencial genético do indi víduo eas condições de vida, determinadas pela sua inserção social". Pretende-se com este estudo identificar a magnitude do efeito dos principais fatores associados à estatura baixa de pré-escolares, em uma população do interior de São Paulo que apresenta padrão de condições de vida um pouco superior à média brasileira e com baixos índices de déficit nutricional.

Material e métodos

Desenho do estudo

Trata-se de um estudo caso-controle exploratório, realizado em 1995, que buscou identificar fatores associados à estatura baixa dos préescolares que freqüentavam a rede pública do Município de Cosmópolis, São Paulo.
Local do estudo

O Município de Cosmópolis, localizado na região nordeste do Estado de São Paulo a 39 quilômetros da cidade de Campinas, contava com 36.646 habitantes, em 1991. A economia do município baseia-se, principalmente, na agroindústria (Prefeitura Municipal de Cosmópolis, 1995). Segundo dados da pesquisa "Qualidade de Vida e Saúde no Município de Cosmópolis 1993-1994", a proporção de analfabetos entre pessoas com 18 anos ou mais, variou de $14,2 \%$ no setor urbano mais carente a $4,2 \%$ no setor urbano de melhor nível sócio-econômico. A proporção de crianças de cinco anos de idade que freqüentavam a pré-escola foi de $37,5 \%$ a $71,4 \%$, segundo o setor de residência, e $64,7 \%$ a $91,7 \%$ das crianças com seis anos de idade freqüentavam as pré-escolas. A média de salários dos trabalhadores do município foi de 327,58 dólares mensais. As famílias eram compostas, em média, por 4,2 membros. Cerca de $70 \%$ das famílias eram proprietárias da casa em que residiam (Barros \& Aoki, 1995).

\section{Levantamento antropométrico}

Os grupos de casos e controles do presente estudo foram obtidos de um levantamento prévio de medidas antropométricas real izado em novembro de 1994, que incluiu o conjunto das crianças que freqüentavam as pré-escolas da rede pública do Município de Cosmópolis. Existiam no município, nesse ano, nove Escolas Municipais de Ensino Integrado freqüentadas por 1.260 alunos, a maioria na faixa de 4 a 7 anos de idade. Destas crianças, 4 foram excluídas por terem menos de 4 anos e 1.201 foram estudadas no inquérito. A perda de $4,37 \%$ decorreu de: faltas consecutivas (51), doença (3) e recusa (1). As medidas antropométricas (peso e estatura) foram coletadas por uma equipe de quatro antropometristas treinados e padronizados segundo as recomendações de Jelliffe (1968). As crianças foram pesadas com indumentária mínima e descalças. O peso foi obtido utilizando balança digital marca Filizolla com capacidade máxima de 150 quilogramas e com precisão de 100 gramas. A estatura foi obtida utilizando uma fita métrica de aço com escala milimétrica e um esquadro de madeira. A leitura da medida era feita atentamente, após a criança deixar a posição sob o esquadro e registrada imediatamente na ficha de levantamento antropométrico.

O índice antropométrico estatura/ idade foi calculado para cada criança segundo os valores em escore Z. Como população de referên- 
cia, adotou-se a do National Center for Health Statistics - NCHS, recomendada pela Organização Mundial de Saúde (WHO, 1995). A avaliação nutricional realizada com esse inquérito detectou que $2,3 \%$ das crianças apresentavam escore $Z<-2,0$ e 16,5\% escore $Z \leq-1,0$ para o índice estatura/idade. A classificação da criança segundo esse índice serviu de base para selecionar os casos e controles do presente estudo.

Definição e seleção de casos e controles

Foram considerados como "casos" as crianças com o índice estatura/idade situado um desvio padrão ou mais abaixo da mediana da população de referência (estatura baixa). As crianças com índice estatura/idade posicionado em um desvio padrão ou mais acima da mediana da população de referência, foram consideradas como "controles". Os seguintes pressupostos nortearam a seleção dos grupos de estudo. Considerou-se que, embora a distribuição da altura/idade na população de estudo fosse semelhante à da população de referência, portanto sem a presença de deficiências significativas de estatura, entre as crianças posicionadas abaixo de - 1 escore $Z$, existiria um percentual de "naturalmente" baixos, mas, provavelmente, um percentual expressivo com algum grau de comprometimento da estatura decorrente de fatores não genéticos. Por outro lado, entre as crianças com o índice superior a um escore Z, a maioria não teria tido prejuízo de sua altura tendo por referência o seu potencial genético de crescimento.

Tamanho da amostra

A amostra foi calculada para detectar uma razão de odds de 2,5, dada uma freqüência relativa de $10 \%$ de um fator de exposição. Considerou-se esse valor por tratar-se de um estudo em que vários fatores de exposi ção seriam analisados e as freqüências de alguns deles na população de origem eram desconhecidas. Definiu-se um poder do estudo (1- $\beta$ ) de $80 \%$ e um erro alfa de $5 \%$. O cálculo da amostra foi baseado em Schlessel man \& Stolley (1982). O tamanho de amostra necessário foi de 151 casos e 151 controles. Acrescentaram-se 9,3\% para compensar possíveis perdas, totalizando 165 crianças em cada grupo. Das 1201 crianças estudadas no levantamento antropométrico, 198 tinham o índice estatura/idade $\leq$ - 1 escore Z e 217 crianças tinham o índice estatura/idade $\geq$ 1 escore Z. Foram selecionadas aleatoriamente 165 crianças para cada grupo.

\section{Questionário e entrevista}

Para obtenção das informações necessárias foram realizadas entrevistas com a mãe ou responsável pela criança. As entrevistas foram realizadas em maio e junho de 1995 nas próprias escolas que as crianças freqüentavam ou nos domicílios, quando necessário. A entrevista foi feita por quatro pesquisadores treinados com o instrumento de coleta de dados.

O questionário aplicado na entrevista continha questões pré-codificadas relativas aos seguintes tópicos: informações sobre a criança (sexo, idade, cor, peso e comprimento ao nascer, ordem de nascimento, antecedentes de morbidade, internação hospitalar e cuidados na infância); composição familiar (identificação do chefe da família, presença dos pais no domicílio, número de moradores, número de irmãos e número de criança menores de cinco anos); características reprodutivas maternas e situação conjugal (número de filhos nascidos vivos, número de filhos mortos, intervalo interpartal, idade materna ao nascimento da criança, estado conjugal, mudança da situação conjugal, número de uniões e separações desde o nascimento da criança); condições do domicílio e saneamento (tipo de casa, condição de ocupação, número de cômodos e de quartos, tipo de equipamentos domésticos, acesso à água potável, rede de esgosto, coleta de lixo); dados sobre migração (naturalidade da criança, número de cidades em que a criança morou, procedência dos pais) e características sócio-econômicas (escolaridade dos pais, renda familiar per capita, ocupação materna e paterna). Quanto à ocupação paterna foram levantados dados sobre posição na ocupação, tempo na ocupação, carga horária semanal de trabaIho, remuneração mensal, número de empregos e ocorrência de desemprego.

Durante as entrevistas foram tomadas medidas de peso e estatura dos pais biológicos das crianças estudadas. Foram utilizados os mesmos instrumentos empregados no levantamento antropométrico realizado com as crianças e as medidas foram tomadas pelo pesquisador principal.

\section{Processamento dos dados}

Os dados do levantamento antropométrico foram processados utilizando o programa CASP Centers for Disease Control Anthropometric Software Package do Epi-Info versão 6.02 (Dean et al., 1994). A digitação foi feita por uma única pessoa e conferida com os dados das fichas de levantamento antropométrico. Os dados do 
questionário foram digitados em um banco elaborado com o programa Epi-Info versão 6.02 (Dean et al., 1994). A digitação das informações foi feita por duas pessoas, sendo que a análise de consistência e correções foram realizadas pelo pesquisador principal.

\section{A nálise dos dados}

$\mathrm{Na}$ análise univariada foram estimados os valores de odds ratio brutas, com intervalo de confiança de $95 \%$ com o uso do programa Epiinfo versão 6.02 (Dean et al., 1994). Por meio do programa SPSS versão 5.02 para Windows, fezse análise de regressão logística múltipla não condicional hierarquizada. Análises hierarquizadas têm sido propostas e utilizadas em estudos brasileiros (Olinto et al., 1993; Fuchs et al., 1996; Victora et al., 1996). Para a análise hierarquizada deste estudo foi seguido o esquema apresentado na Figura 1.

Figura 1

Modelo de análise de estatura de pré-escolares.

Nível sócio-econômico

Características da família

e reprodutivas materna

Sexo, idade, cor

Estatura dos pais

Peso e comprimento ao nascer

Estatura de pré-escolares

\section{Resultados}

Das 330 crianças sorteadas para o estudo, a entrevista pôde ser realizada com 307, sendo 156 casos e 151 controles. Os motivos de perdas foram: mudança (12), não localização de endereços (9) e doença do responsável (2). Os resultados da análise univariada estão apresentados nos seguintes tópicos: variáveis indicadoras do nível sócio-econômico, variáveis relativas às características da família e maternas, variáveis do domicílio, variáveis antropométricas e sexo. Não foram incluídos nas tabelas os resultados das variáveis que não apresentaram associação estatisticamente significativa com a estatura baixa.

A Tabela 1 apresenta os resultados referentes às variáveis sócio-econômicas. Mostraram-se associadas com a presença de estatura baixa a escolaridade da mãe, a renda familiar per capita e algumas variáveis relacionadas à ocupação do pai: número de empregos desde o nascimento da criança, tempo de ocupação no emprego atual e remuneração mensal. Pré-escolares cujas rendas familiares per capita foram menores que 1/2 salário mínimo (SM) apresentaram chance 4,4 vezes maior de estatura baixa que aqueles de famílias com renda superior a 2,5 SM.

As variáveis relativas à família e reprodutivas maternas que se apresentaram estatisticamente significativas na análise univariada foram: número de pessoas que moram no domicílio, número de irmãos, número de crianças menores de cinco anos, naturalidade da criança, número de filhos nascidos vivos e intervalo interpartal. Crianças cujas mães tinham tido quatro ou mais filhos apresentaram chance 3,5 vezes maior de estatura baixa que aquelas cujas mães tinham tido apenas um filho. A chance de estatura baixa foi maior nas crianças que tinham nascido após período interpartal menor ou igual a 24 meses (Tabela 2).

O tipo de acabamento da casa, número de cômodos no domicílio, número de quartos e número de equipamentos domésticos mostraram-se associados estatisticamente com a estatura baixa de pré-escolares (Tabela 3). O número de equipamentos foi calculado com base na presença ou não no domicílio dos equipamentos que haviam mostrado isoladamente associação com a baixa estatura: geladeira, freezer, lava-roupa e telefone. Crianças com um ou nenhum desses equipamentos no domicílio apresentaram chance 5,4 vezes maior de estatura baixa que as que tinham três ou quatro equipamentos em suas casas.

O sexo, o peso e o comprimento ao nascer da criança e a estatura dos pais apresentaram- 
Tabela 1

Distribuição dos casos, controles, odds ratio bruta e respectivo intervalo de confiança de $95 \%$ segundo variáveis sócio-econômicas. Cosmópolis, SP,1995.

\begin{tabular}{|c|c|c|c|c|c|c|}
\hline Variável & Caso & Controle & Total & ORbruta & IC $95 \%\left(O R_{b}\right)$ & $p\left(\chi^{2}\right)$ \\
\hline \multicolumn{7}{|l|}{ Escolaridade da mãe } \\
\hline Primário completo e mais & 21 & 42 & 63 & 1 & - & \\
\hline A té primário incompleto & 131 & 107 & 238 & 2,5 & $1,3-4,6$ & \\
\hline Total & 152 & 149 & $301 *$ & - & - & 0,003 \\
\hline \multicolumn{7}{|c|}{ Renda familiar per capita (SM)** } \\
\hline 2,51 e mais & 17 & 42 & 59 & 1 & - & \\
\hline $2,01-2,50$ & 11 & 17 & 28 & 1,6 & $0,6-4,7$ & \\
\hline $1,51-2,00$ & 21 & 16 & 37 & 3,2 & $1,3-8,4$ & \\
\hline $1,01-1,50$ & 35 & 20 & 55 & 4,3 & $1,8-10,3$ & \\
\hline $0,51-1,00$ & 42 & 39 & 81 & 2,7 & $1,2-5,8$ & \\
\hline$\leq 0,50$ & 30 & 17 & 47 & 4,4 & $1,8-10,8$ & \\
\hline Total & 156 & 151 & 307 & - & - & 0,001 \\
\hline \multicolumn{7}{|l|}{ Tempo de ocupação do pai } \\
\hline$\geq 5$ anos & 44 & 63 & 107 & 1 & - & \\
\hline$<5$ anos & 84 & 68 & 152 & 1,8 & $1,0-3,0$ & \\
\hline Total & 128 & 131 & $259 *$ & - & - & 0,034 \\
\hline \multicolumn{7}{|c|}{ Remuneração mensal do pai (SM)** } \\
\hline 8,01 e mais & 24 & 43 & 67 & 1 & - & \\
\hline $4,01-8,00$ & 62 & 46 & 108 & 2,4 & $1,2-4,8$ & \\
\hline Até 4,00 & 42 & 39 & 81 & 1,9 & $0,9-4,0$ & \\
\hline Total & 128 & 128 & $256^{*}$ & - & - & 0,020 \\
\hline \multicolumn{7}{|l|}{ № de empregos do pai } \\
\hline 1 & 37 & 57 & 94 & 1 & - & \\
\hline Mais que 1 & 92 & 73 & 165 & 2,0 & $1,2-3,5$ & \\
\hline Total & 129 & 130 & $259 *$ & & & 0,012 \\
\hline
\end{tabular}

* excluídas as crianças com informação ignorada.

** SM = salário mínimo.

se estatisticamente associados com a estatura baixa (Tabela 4).

Entre as variáveis que na análise univariada não mostraram associação com a baixa estatura encontravam-se: a idade, a presença de morbidade e o número de internações hospitalares desde o nascimento da criança. Notou-se um aumento nos valores das odds ratio segundo a idade da criança, mas sem significância estatística. A informação sobre morbidade foi obtida perguntando-se se a criança apresentava algum problema de saúde (grave e freqüente) ou deficiência física. Não foram feitas perguntas específicas sobre patologias associadas à desnutrição. Quanto à variável internação hospitalar verificou-se um aumento da chance de estatura baixa com o aumento do número de internações, mas sem significância estatística.
A odds ratio para uma internação foi de 1,2 (IC $95 \%=[0,73-2,1])$ e para duas internações foi de $1,45$ (IC $95 \%=[0,70-3,0])$, em comparação com nenhuma internação.

Os resultados da análise multivariada hierarquizada estão apresentados na Tabela 5. Entre as variáveis sócio-econômicas, permaneceram como estatisticamente significativas a escolaridade materna (OR $=2,1$ IC $95 \%=[1,1-3,8]$ ) e a renda familiar per capita. A chance da criança ter estatura baixa foi 3,4 vezes maior (IC $95 \%=[1,5-8,0])$ nas famílias com renda familar per capita menor ou igual a 0,5 SM, em comparação com aquelas que tinham renda superior a $2,51 S M$.

Das variáveis relacionadas à familia e reprodutivas maternas bem como às relacionadas ao domicílio, permaneceram significativas 
Tabela 2

Distribuição dos casos, controles, odds ratio bruta e respectivo intervalo de confiança de $95 \%$ segundo características da família e reprodutivas maternas. Cosmópolis, SP, 1995.

\begin{tabular}{|c|c|c|c|c|c|c|}
\hline Variável & Caso & Controle & Total & OR bruta & IC $95 \%\left(O R_{b}\right)$ & $p\left(\chi^{2}\right)$ \\
\hline \multicolumn{7}{|l|}{ No de pessoas } \\
\hline$<4$ & 16 & 28 & 44 & 1 & - & \\
\hline 4 & 43 & 52 & 95 & 1,4 & $0,6-3,2$ & \\
\hline 5 & 41 & 42 & 83 & 1,7 & $0,8-3,9$ & \\
\hline 6 e mais & 56 & 29 & 85 & 3,4 & $1,7-7,8$ & \\
\hline Total & 156 & 151 & 307 & - & - & 0,005 \\
\hline \multicolumn{7}{|c|}{ № de crianças $<5$ anos } \\
\hline Nenhuma & 81 & 99 & 180 & 1 & - & \\
\hline 1 e mais & 75 & 52 & 127 & 1,8 & $1,1-2,9$ & \\
\hline Total & 156 & 151 & 307 & - & - & 0,020 \\
\hline \multicolumn{7}{|l|}{ № de irmãos } \\
\hline Até 1 & 76 & 100 & 176 & 1 & - & \\
\hline 2 & 41 & 40 & 81 & 1,4 & $0,8-2,4$ & \\
\hline 3 e mais & 39 & 11 & 50 & 4,7 & $2,1-10,4$ & \\
\hline Total & 156 & 151 & 307 & - & - & $<0,001$ \\
\hline \multicolumn{7}{|c|}{ Naturalidade da criança } \\
\hline São Paulo & 135 & 144 & 279 & 1 & - & \\
\hline O utros estados & 21 & 7 & 28 & 3,2 & $1,2-8,6$ & \\
\hline Total & 156 & 151 & 307 & - & - & 0,013 \\
\hline \multicolumn{7}{|c|}{ № de filhos nascidos vivos* } \\
\hline 1 & 24 & 36 & 60 & 1 & - & \\
\hline 2 & 42 & 53 & 95 & 1,2 & $0,6-2,5$ & \\
\hline 3 & 38 & 31 & 69 & 1,8 & $0,9-3,9$ & \\
\hline 4 e mais & 42 & 18 & 60 & 3,5 & $1,5-8,0$ & \\
\hline Total & 146 & 138 & 284 & - & - & $<0,001$ \\
\hline \multicolumn{7}{|c|}{ Intervalo interpartal (meses)** } \\
\hline$\geq 36$ & 39 & 48 & 87 & 1 & - & \\
\hline $25-35$ & 16 & 10 & 26 & 1,9 & $0,7-5,3$ & \\
\hline$\leq 24$ & 32 & 15 & 47 & 2,6 & $1,2-5,3$ & \\
\hline Total & 87 & 73 & 160 & - & - & 0,026 \\
\hline
\end{tabular}

* mães biológicas.

** excluídas primigestas e informação ignorada.

o número de pessoas que moram no domicílio e o número de equipamentos domésticos, quando ajustadas entre si e pelas variáveis do bloco anterior (escolaridade materna e renda familiar per capita).

Mantiveram-se altamente associadas com a estatura baixa da criança, após ajuste com as variáveis dos blocos anteriores, o comprimento ao nascer e as estaturas materna e paterna.

A odds ratio do sexo masculino em relação ao feminino, após o ajuste, atingiu 2,1 (IC 95\% = $[1,1-3,9])$.

\section{Discussão}

No presente estudo, a perda em relação às crianças sorteadas foi de 5,5\% nos casos e 8,6\% nos controles, não devendo afetar os resultados obtidos. Das entrevistas realizadas, 92,5\% foram com as mães ( $18,5 \%$ das vezes acompanhadas pelo pai ), $5,2 \%$ com o pai e $2,3 \%$ foram realizadas com outro membro da família (avó ou tia). A duração mediana das entrevistas foi de 15 minutos ( $m$ ínimo = 5 minutos e máximo $=35$ minutos). 0 percentual de informa- 
Distribuição dos casos, controles, odds ratio bruta e respectivo intervalo de confiança de $95 \%$, segundo variáveis relacionadas ao domicílio. Cosmópolis, SP, 1995.

\begin{tabular}{|c|c|c|c|c|c|c|}
\hline Variável & Caso & Controle & Total & OR bruta & IC $95 \%\left(O R_{b}\right)$ & $p\left(\chi^{2}\right)$ \\
\hline \multicolumn{7}{|c|}{ Tipo de acabamento da casa } \\
\hline completo & 85 & 104 & 189 & 1 & - & \\
\hline incompleto & 71 & 47 & 118 & 1,8 & $1,1-3,0$ & \\
\hline Total & 156 & 151 & 307 & - & - & 0,013 \\
\hline \multicolumn{7}{|c|}{ № de cômodos } \\
\hline 6 e mais & 47 & 70 & 117 & 1 & - & \\
\hline 5 & 40 & 41 & 81 & 1,4 & $0,8-2,7$ & \\
\hline 4 & 31 & 17 & 48 & 2,7 & $1,3-5,8$ & \\
\hline$<4$ & 38 & 23 & 61 & 2,5 & $1,2-4,9$ & \\
\hline Total & 156 & 151 & 307 & - & - & 0,007 \\
\hline \multicolumn{7}{|l|}{$\mathrm{N}$ o de quartos } \\
\hline 3 e mais & 41 & 57 & 98 & 1 & - & \\
\hline 2 & 63 & 61 & 124 & 1,4 & $0,8-2,5$ & \\
\hline 1 & 52 & 33 & 85 & 2,2 & $1,2-4,1$ & \\
\hline Total & 156 & 151 & 307 & - & - & 0,033 \\
\hline \multicolumn{7}{|c|}{ № de equipamentos domésticos } \\
\hline 3 a 4 & 13 & 42 & 55 & 1 & & \\
\hline 2 & 103 & 85 & 188 & 3,9 & $1,9-8,2$ & \\
\hline$\leq 1$ & 40 & 24 & 64 & 5,4 & $2,2-13,1$ & \\
\hline Total & 156 & 151 & 307 & - & - & $<0,001$ \\
\hline
\end{tabular}

ções ignoradas foi mínimo e mesmo inexistente para a maior parte das variáveis estudadas. Atingiu valores importantes nas variáveis relacionadas à ocupação do pai (16\%) e comprimento ao nascer (21,8\%).

As variáveis número de filhos nascidos vivos e intervalo interpartal foram obtidas somente das mães biológicas (casos $=146$ e controles $=138$ ), que residiam no domicílio da criança. A estatura da mãe e do pai foi tomada de todos pais biológicos que compareceram à pré-escola, independente de estarem morando no domicílio da criança estudada.

Quanto aos fatores estudados, constatouse neste estudo que as crianças cujas mães tinham apenas primário incompleto apresentaram maior chance $\left(\mathrm{OR}_{\text {ajustada }}=2,1\right.$; IC $95 \%=$ [1,1-3,8]) de ter estatura baixa.

A escolaridade materna como um dos determinantes do crescimento infantil tem sido freqüentemente evidenciada na literatura. Bobák et al. (1994) verificaram em crianças com cinco anos de idade que aquelas cujas mães tinham apenas educação primária apresentavam média de estatura 0,3 desvios padrão abai- xo da média das filhas de mães com nível universitário. Mães analfabetas apresentaram risco quase três vezes maior de ter um filho com déficit de estatura/ídade que mães com quatro ou mais anos de escolaridade, segundo estudo realizado em áreas urbanas da região nordeste, em crianças menores de cinco anos (Cardoso, 1995).

A importância da escolaridade materna na proteção à saúde da criança decorre dos cuidados preventivos e curativos que a mãe realiza com mais propriedade, por ter mais conhecimento e/ ou acesso a serviços. A forma como a mulher dedica sua atenção aos filhos, tanto diretamente como delegando a terceiros este cuidado, é influenciada pelo nível de escolaridade (Cardoso, 1995).

A influência que a renda familiar exerce sobre o crescimento infantil é evidenciada em vários trabalhos (Johnson \& Rogers, 1993; Miller \& Korenman, 1994; Monteiro el al., 1995). Neste estudo a chance de estatura baixa nos préescolares foi maior nos estratos de renda familiar mais baixos ainda que sem apresentar um gradiente integralmente consistente. Após o 
Tabela 4

Distribuição dos casos, controles, odds ratio bruta e respectivo intervalo de confiança de $95 \%$, segundo sexo e variáveis antropométricas. Cosmópolis, SP, 1995.

\begin{tabular}{|c|c|c|c|c|c|c|}
\hline Variável & Caso & Controle & Total & OR bruta & IC $95 \%\left(O R_{b}\right)$ & $p\left(\chi^{2}\right)$ \\
\hline \multicolumn{7}{|l|}{ Sexo } \\
\hline feminino & 62 & 78 & 140 & 1 & - & \\
\hline masculino & 94 & 73 & 167 & 1,6 & $1,0-2,6$ & \\
\hline Total & 156 & 151 & 307 & - & - & 0,048 \\
\hline \multicolumn{7}{|c|}{ Peso ao nascer (gr) } \\
\hline$>3500$ & 30 & 61 & 91 & 1 & - & \\
\hline $3000-3500$ & 54 & 56 & 110 & 1,9 & $1,1-3,6$ & \\
\hline $2500-2999$ & 40 & 18 & 58 & 4,5 & $2,1-9,8$ & \\
\hline$<2500$ & 16 & 6 & 22 & 5,4 & $1,7-17,5$ & \\
\hline Total & 140 & 141 & $281^{*}$ & - & - & $<0,001$ \\
\hline \multicolumn{7}{|c|}{ Comprimento ao nascer $(\mathrm{cm})$} \\
\hline 50 e mais & 45 & 89 & 134 & 1 & - & \\
\hline $48-49$ & 39 & 34 & 73 & 2,2 & $1,2-4,1$ & \\
\hline$<48$ & 27 & 6 & 33 & 8,9 & $3,2-26,1$ & \\
\hline Total & 111 & 129 & $240 *$ & - & - & $<0,001$ \\
\hline \multicolumn{7}{|c|}{ Estatura da mãe $(\mathrm{cm})^{* *}$} \\
\hline$>156,6$ & 44 & 103 & 147 & 1 & - & \\
\hline até 156,6 & 106 & 35 & 141 & 7,1 & $4,1-12,4$ & \\
\hline Total & 150 & 138 & $288^{*}$ & - & - & $<0,001$ \\
\hline \multicolumn{7}{|c|}{ Estatura do pai $(\mathrm{cm})^{* *}$} \\
\hline$>169,8$ & 35 & 87 & 122 & 1 & - & \\
\hline até 169,8 & 90 & 31 & 121 & 7,2 & $3,9-13,3$ & \\
\hline Total & 125 & 118 & $243 *$ & - & - & $<0,001$ \\
\hline
\end{tabular}

* excluídas as crianças com informação ignorada.

** os pontos de corte 156,6 e 169,8 representam as médias obtidas na amostra.

controle pela variável escolaridade materna, as odds ratio continuaram significativas.

A forma de inserção do chefe da família no mercado de trabalho é uma condição importante, pois determina as possibilidades e limites de acesso a bens e serviços pelas famílias. Neste estudo, algumas variáveis relacionadas à ocupação do pai como tempo de ocupação no emprego atual, remuneração mensal e número de empregos desde o nascimento da criança, mostraram associação apenas na análise univariada, não permanecendo significativa na presença de escolaridade materna e renda familiar per capita.

$O$ acesso a bens e equipamentos domésticos constitui medida do poder aquisitivo (Barros \& Barros, 1994; Cardoso, 1995). Além disso, alguns equipamentos domésticos são importantes na conservação dos alimentos e outros facilitam o acesso a informações, diferenciando o estilo de vida das famílias. Observou-se uma relação inversa entre a diminuição do número de equipamentos domésticos e a chance de estatura baixa nos pré-escolares.

As condições de moradia, seja o seu tamanho ou o tipo de acabamento estiveram associadas com estatura baixa somente na análise univariada. Estudo realizado com escolares também verificou que as crianças que moravam em barracos ou em casas de tijolos precariamente construídas eram mais baixas que as que residiam em casas de melhor nível (Barros $\&$ Barros, 1994)

A literatura tem evidenciado a associação entre a alta paridade - menor disponibilidade do tempo materno, diminuição do aleitamento materno, distribuição intradomiciliar reduzida dos alimentos - com o retardo estatural (Cardoso, 1995).

A observação, neste estudo, de maior ocorrência de estatura baixa no sexo masculino, 
Tabela 5

Resultados da análise de regressão logística múltipla hierarquizada. Cosmópolis, SP. 1995.

\begin{tabular}{|c|c|c|c|c|c|}
\hline Modelo & OR bruta & O Rajustada & IC95\% $\left(\mathrm{OR}_{\mathrm{a}}\right)$ & $\mathrm{p}$ (categoria) & p (variável) \\
\hline \multicolumn{6}{|l|}{ Fatores sócio-econômicos* } \\
\hline \multicolumn{6}{|l|}{ Escolaridade da mãe } \\
\hline Primário completo e mais & 1 & 1 & - & - & 0,0695 \\
\hline Até primário incompleto & 2,5 & 2,1 & {$[1,1 ; 3,8]$} & 0,0226 & \\
\hline \multicolumn{6}{|l|}{ Renda familiar per capita } \\
\hline 2,51 e mais & 1 & 1 & - & - & 0,0092 \\
\hline $2,01-2,50$ & 1,6 & 1,4 & {$[0,5 ; 3,6]$} & 0,5284 & \\
\hline $1,51-2,00$ & 3,2 & 2,8 & {$[1,2 ; 6,7]$} & 0,0222 & \\
\hline $1,01-1,50$ & 4,3 & 3,9 & {$[1,8 ; 8,7]$} & 0,0008 & \\
\hline $0,51-1,00$ & 2,7 & 2,2 & {$[1,0 ; 4,6]$} & 0,0359 & \\
\hline$\leq 0,50$ & 4,4 & 3,4 & {$[1,5 ; 8,0]$} & 0,0042 & \\
\hline
\end{tabular}

Características da família e domicílio**

№ de pessoas que moram no domicílio

$\begin{array}{lllcrl}<4 & 1 & 1 & - & & 0,0239 \\ 4 & 1,4 & 1,8 & {[0,8 ; 4,0]} & 0,1517 & \\ 5 & 1,7 & 1,7 & {[0,7 ; 3,9]} & 0,2115 & \\ 6 \text { e mais } & 3,4 & 3,7 & {[1,5 ; 9,0]} & 0,0039 & \\ & & & & & 0,0025 \\ \text { № de equipamentos domésticos } & 1 & 1 & - & - & 0,0016 \\ \text { 3 e mais } & 3,9 & 3,4 & {[1,6 ; 7,1]} & 0,0012\end{array}$

Sexo e variáveis antropométricas***

\begin{tabular}{|c|c|c|c|c|c|}
\hline \multicolumn{6}{|l|}{ Sexo } \\
\hline feminino & 1,0 & 1,0 & - & - & \multirow[t]{2}{*}{0,005} \\
\hline masculino & 1,6 & 2,1 & {$[1,1 ; 3,9]$} & 0,0233 & \\
\hline \multicolumn{6}{|c|}{ Comprimento ao nascer } \\
\hline 50 e mais & 1,0 & 1,0 & - & - & \multirow[t]{3}{*}{0,0005} \\
\hline $48-49$ & 2,2 & 2,8 & {$[1,3 ; 6,1]$} & 0,0087 & \\
\hline$<48$ & 8,9 & 7,4 & {$[2,3 ; 23,7]$} & 0,0008 & \\
\hline \multicolumn{6}{|c|}{ Estatura da mãe $(\mathrm{cm})$} \\
\hline$>156,6$ & 1,0 & 1,0 & - & - & \multirow[t]{2}{*}{$<0,001$} \\
\hline até 156,6 & 7,1 & 5,9 & {$[3,1 ; 11,0]$} & $<0,001$ & \\
\hline \multicolumn{6}{|c|}{ Estatura do pai $(\mathrm{cm})$} \\
\hline$>169,8$ & 1,0 & 1,0 & - & - & \multirow[t]{2}{*}{$<0,001$} \\
\hline até 169,8 & 7,2 & 4,2 & {$[2,1 ; 8,6]$} & $<0,001$ & \\
\hline
\end{tabular}

* Ajustada pela outra variável, incluída no modelo.

** Ajustadas pelas variáveis escolaridade da mãe, renda familiar per capita e pela outra variável incluída no modelo

*** Ajustadas pelas variáveis escolaridade da mãe, renda familiar per capita, número de equipamentos domésticos, número de pessoas que moram no domicílio e demais variáveis que constam no modelo. 
concorda com o relatado na literatura (Tanner, 1987; Barros et al., 1990).

Entre as famílias que vivem em situações sócio-econômicas desfavoráveis é mais freqüente o nascimento de crianças com baixo peso e com déficit de comprimento (Miller \& Korenman, 1994). Uma precária situação econômica tende a perdurar após o nascimento da criança, agravando o déficit antropométrico observado ao nascer. Por outro lado, recémnascidos pequenos, freqüentemente, apresentam dificuldades de amamentação e tendem a ter mais episódios de doenças, acentuando o comprometimento da estatura. Após ajuste pelas demais variáveis o comprimento ao nascer foi a variável que apresentou a associação mais forte com a baixa estatura no pré-escolar. Crianças com menos de $48 \mathrm{~cm}$ de comprimento ao nascer apresentaram chance ajustada 7,4 vezes maior de estatura baixa que as nascidas com $50 \mathrm{~cm}$ ou mais.

Uma associação estatística forte entre estatura dos pais e estatura baixa dos filhos foi também observada no presente estudo.

Algumas publicações têm mostrado que entre crianças de nível sócio-econômico alto, quando o potencial de crescimento é totalmente expresso, a estatura baixa dos pais é um bom preditor da estatura das crianças (Amigo \& Bustos, 1995; Voss et al., 1998). Nos níveis sócio-econômicos baixos, outros fatores, além do potencial genético, podem explicar a ocorrência de estatura baixa na população infantil. Estudo realizado com escolares da área rural do Chile constata que as variáveis estatura do pai ( $O R=4,98)$, estatura da mãe $(O R=4,64)$, subnutrição dos pais (OR $=4,53$ ) e peso ao nascer insuficiente ( $O R=3,23$ ) foram associadas com estatura baixa dos escolares ( $\leq 1$ escore $z$ ) (Amigo \& Bustos, 1995).

Em síntese, a análise múltipla hierarquizada indicou neste estudo como fatores sócioeconômicos associados à estatura baixa: a escolaridade materna e renda familiar per capita. Como variáveis relativas à família e domicílio: o número de pessoas que moram no domicílio e o número de equipamentos domésticos. Mostraram-se, ainda associados, o sexo e as variáveis antropométricas: comprimento ao nascer e estatura dos pais.

Verifica-se então que mesmo em uma população de escolares em que a deficiência nutricional, detectada pelos índices antropométricos usuais, é mínima ou inexistente, pois apresenta os mesmos percentuais abaixo de - 1 ou de - 2 desvios padrão que a população de referência, é possível evidenciar o efeito de variáveis sócio-econômicas na estatura de pré-escolares. Também é possível considerar que a relação entre os efeitos das variáveis sociais e daquelas marcadoras do potencial genético (como a estatura de pais) é condicionada pelo nível de desenvolvimento econômico e social da população estudada. A população do presente estudo apresenta razoável padrão de condições de vida frente à média brasileira e apresenta baixos índices de déficit nutricional. As ORs relativas às variáveis antropométricas apresentaram-se mais elevadas que as relativas às variáveis sociais. Seria possível considerar que quanto mais elevado o nível sócio-econômico de populações ou subgrupos populacionais, maior o potencial de preditor da estatura dos pais em relação à estatura dos filhos.

\section{Referências}

AMIGO, H. \& BUSTOS, P., 1995. Risk factors of short stature in Chilean school children from rural areas of high social vulnerability. Archivos Latinoamericanos de Nutrición, 45:97-102.

AMIGO, H.; BUSTOS, P. \& RADRIGAN, M. E., 1997. Is there a relationship between parent's short height and their children's? Revista Médica de Chile, 125: 863-868.

BARROS, A. A.; BARROS, M. B. A.; MAUDE, G. H.; ROSS, D. A.; DAVIES, P. S. W. \& PREECE, M. A., 1990. Evaluation of the nutritional status of 1styear shoool children in Campinas, Brazil. Annals of Tropical Paediatrics, 10:75-84. 
BARROS, M. B. A. \& BARROS, A. A. F., 1994. Fatores que influenciam a estatura de escolares em área urbana do Brasil. In: 2nd International Congress of Nutrition in Pediatrics, Annals. Lisboa: International Society of Nutritional Pediatrics/Sociedade Portuguesa de Pediatria.

BARROS, M. B. A. \& AOKI, E. A., 1995. Pesquisa Saúde eQualidade deVida em Cosmópolis. Relatório Final. Campinas, São Paulo: Departamento de Medicina Preventiva e Social, Universidade Estadual de Campinas. (mimeo.)

BICUDO-ZEFERINO, A. M., 1992. Crescimento nos Dois Primeiros Anos deVida - Estudo em Crianças de Dois Grupos Sociais Distintos. Tese de Doutorado, Campinas: Faculdade de Ciências M édicas, Universidade Estadual de Campinas.

BOBÁK, M.; BOHUMIR, K.; LEON, D. A.; DANOVÁ, J. \& MARM OT, M., 1994. Socioeconomic factors on height of preschool children in the Czech Republic. American Journal of Public Health, 84:11671170.

CARDOSO, M. A. A., 1995. Cuidado Infantil e Desnutrição de Pré-Escolares: Regi ão Nordeste e Sul do Brasil. Tese de Doutorado, São Paulo: Faculdade de Saúde Pública, Universidade de São Paulo.

DEAN, A. G.; DEAN, J. A.; BURTON, A. H. \& DICHER, R. C., 1994. Epi Info, Version 6: A Word Processing, Database, and Statistics Program for Epidemiology on Micro-Computers. Atlanta: Centers for Disease Control and Prevention.

FUCHS, S. C.; VICTORA, C. G. \& FACHEL, J., 1996. Modelo hierarquizado: Uma proposta de modelagem aplicada à investigação de fatores de risco para diarréia grave. Revista de Saúde Pública, 30: 168-178.

GOLDSTEIN, H., 1971. Factors infuencing the height of seven year old children - Results from the National Child Development Study. Human Biology, 43:92-111.

GUILLIFORD, M. C.; CHINN, S. \& RONA, R. J., 1991. Social enviroment and height: England and Scotland 1987 and 1988. Archives of Disease in Childhood, 66:235-240.

JELLIFFE, D. B., 1968. Evaluación del Estado de Nutrición dela Comunidad. Série de Monografias, 53. Ginebra: Organización Mundial de la Salud.
JOHNSON, F. C. \& ROGERS, B. L., 1993. Children's nutritional status in female-headed households in the Dominican Republic. Social Scienceand Medicine, 37:1293-1301.

LINDGREN, G. W. \& CERNERUD, L., 1992. Physical growth and socioeconomic background of Stockholm schoolchildren born in 1933-63. Annals of Human Biology, 19:1-16.

MILLER, J. E. \& KORENMAN, S., 1994. Poverty and children's nutritional status in the United States. American Journal of Epidemiology, 3:233-242.

MONTEIRO, C. A.; BENÍCIO, M. H. D.; IUNES, R. F.; GOUVEIA, N. C. \& CARDOSO, M. A. A., 1995. Evolução da desnutrição infantil. In: Vel hos e Novos Males da Saúde no Brasil (C. A. Monteiro, org.), pp. 93-114, São Paulo: Editora Hucitec/ Núcleo de Pesquisas Epidemiológicas em Nutrição e Saúde, Universidade de São Paulo.

OLINTO, M. T. A.; VICTORA, C. G.; BARROS, F. C. \& TOMASI, E., 1993. Determinantes da desnutrição infantil em uma população de baixa renda: Um modelo de análise hierarquizado. Cadernos de SaúdePública, 9:14-27.

PREFEITURA MUNICIPAL DE COSMÓPOLIS., 1995. Informativo. Cosmópolis: Prefeitura Municipal. (mimeo.)

SCHLESSELM AN, J. J. \& STOLLEY, P. D., 1982. CaseControl Studies. New York: Oxford University Press.

TANNER, J. M., 1987. Growth as a mirror of the conditions of society: Secular trends and class distinctions. Acta Paediatric Japanese, 29:96-103.

VICTORA, C. G.; HUTTLY, S. R.; FUCHS, S. C. \& OLINTO, M. T. A., 1996. The role of conceptual frameworks in epidemiological analysis: A hierarchial approach. International Journal of Epidemiology, 6:1-4.

VOSS, L. D.; MULLIGAN, J. \& BETTS, P. R., 1998. Short stature at school entry - An index of social deprivation? Child Care Health Development. 24:145156.

WHO (World Health Organization), 1995. Expert Committee. Physical Status: TheUseand Interpretation of Antropomethry. Technical Report Series 854. Geneva: WHO. 